\title{
Difference numerical solutions for time-space fractional advection diffusion equation
}

\author{
Fangfang Zhang ${ }^{1 *}$, Xiaoyang Gao ${ }^{1}$ and Zhaokun Xie
}

\section{"Correspondence: \\ zhangff1986@163.com \\ 'School of Electrical Engineering, Zhengzhou University, Zhengzhou, China}

\begin{abstract}
In this paper, a time-space fractional advection diffusion equation is considered for the natural extension of the convection diffusion equation. An explicit difference scheme and an implicit difference scheme are presented. The stability and convergence of the two difference schemes are discussed. It is shown that the explicit difference scheme is conditionally stable and convergent, and the implicit difference scheme is unconditionally stable and convergent. The convergence order of the two methods is $O(\tau+h)$.

Keywords: Fractional convection-diffusion equation; Time-space fractional derivative; Explicit difference scheme; Implicit difference scheme; Stability; Convergence
\end{abstract}

\section{Introduction}

The research and application of fractional differential equations have attracted the attention of many scholars in the last few decades. Fractional order differential equations are generalizations of classical differential equations. They are used to describe the models and phenomena in some natural physical processes, chemical reactions, water pollution, financial markets, and so on [1-3]. In order to get the solutions of these equations, many methods had been developed and used, such as analytical methods, approximation methods, difference methods, etc. [4-10].

The fractional order convection diffusion equations are the generalization of the integral order convection diffusion equations. They are produced in the process of some anomalous diffusion. For general fractional advection diffusion equation, it is hard to get the accurate result. And the numerical method is important and useful in dealing with fractional differential equations. Yu studied the implicit difference approximation of the time fractional reaction diffusion equation in [11]. Zhuang [12] and Tan [13] separatively studied the explicit and implicit difference schemes for time-space fractional reactiondiffusion equations, and the stability and convergence were discussed. Based on the integral transform method, Povstenko [14] and Zhang [15] obtained the analytical results of time-fractional diffusion-wave equation with a source term in cylindrical coordinates. The Jacobi collocation method for solving a special kind of the fractional advection diffusion equation with a nonlinear source term was used in [16]. Finite element multigrid

(c) The Author(s) 2019. This article is distributed under the terms of the Creative Commons Attribution 4.0 International License (http://creativecommons.org/licenses/by/4.0/), which permits unrestricted use, distribution, and reproduction in any medium, provided you give appropriate credit to the original author(s) and the source, provide a link to the Creative Commons license, and indicate if changes were made. 
method for multi-term time fractional advection diffusion equations was given in [17]. Two approximate results in two different generalizations of the space-time-fractional advection diffusion equation were discussed in [18]. The fundamental solutions were obtained by the Laplace transform and Fourier transform, and the numerical results were also obtained. A fully implicit finite difference scheme based on extended cubic B-splines for time fractional advection diffusion equation was obtained in [19]. This study [20] was concerned with the mixed initial boundary value problem for a dipolar body in the context of the thermoelastic theory, and the Hölder-type stability was also discussed. It is well known that fractional operators are nonlocal and hence independent of the used numerical methods. In order to have an acceptable computational cost, the structure of Toeplitz or Toeplitz-times-diagonal type were exploited and discussed in [21, 22].

In this paper, based on the finite difference method, we discuss a time-space fractional advection diffusion equation, where the time term, the advection term, and the diffusion term are all fractional order derivatives. An explicit difference scheme and an implicit difference scheme are obtained. Then the stability and convergence of the two difference schemes are discussed. The paper is organized as follows. The time-space fractional advection diffusion model is given in Sect. 2. The explicit difference scheme is presented in Sect. 3. In Sect. 4, the implicit difference scheme is discussed. The conclusions are presented in Sect. 5.

\section{Problem formulation}

In this paper, the following time-space fractional advection diffusion equation is considered:

$$
\frac{\partial^{\alpha} u(x, t)}{\partial t^{\alpha}}=-b(x) \frac{\partial^{\gamma} u(x, t)}{\partial x^{\gamma}}+a(x) \frac{\partial^{\beta} u(x, t)}{\partial x^{\beta}}+s(x, t)
$$

where $0 \leq x \leq L, 0 \leq t \leq T$, and $L, T$ are any given finite constants. And the initial value condition and the boundary value condition are as follows:

$$
\begin{aligned}
& u(x, 0)=f(x), \quad 0 \leq x \leq L, \\
& u(0, t)=u(L, t)=0, \quad t>0,
\end{aligned}
$$

in which $a(x)>0, b(x)>0,0<\alpha \leq 1,1<\beta \leq 2,0<\gamma \leq 1$ and $f(x)$ is one given function.

$\frac{\partial^{\alpha} u(x, t)}{\partial t^{\alpha}}$ and $\frac{\partial^{\gamma} u(x, t)}{\partial x^{\gamma}}$ are defined as the Caputo fractional derivative

$$
\begin{aligned}
& \frac{\partial^{\alpha} u(x, t)}{\partial t^{\alpha}}=\frac{1}{\Gamma(1-\alpha)} \int_{0}^{t}(t-\eta)^{-\alpha} \frac{\partial u(x, \eta)}{\partial \eta} d \eta, \\
& \frac{\partial^{\gamma} u(x, t)}{\partial x^{\gamma}}=\frac{1}{\Gamma(1-\gamma)} \int_{0}^{x}(x-\zeta)^{-\gamma} \frac{\partial u(\zeta, t)}{\partial \zeta} d \zeta .
\end{aligned}
$$

$\frac{\partial^{\beta} u(x, t)}{\partial x^{\beta}}$ is defined as the Riemann-Liouville fractional derivative

$$
\frac{\partial^{\beta} u(x, t)}{\partial x^{\beta}}=\frac{1}{\Gamma(2-\beta)} \frac{\partial^{2}}{\partial x^{2}} \int_{0}^{x} \frac{u(\xi, t)}{(x-\xi)^{\beta-1}} d \xi .
$$


For the normal situation, it is very difficult to get an analytical solution of equations (2.1)-(2.3). Let us consider the numerical solution of the equations based on the difference method in the following sections.

\section{Explicit difference scheme}

Let $t_{k}=k \tau, k=0,1,2, \ldots, n ; x_{i}=i h, i=0,1,2, \ldots, m$, where $\tau=\frac{T}{n}$ and $h=\frac{L}{m}$ represent the time step and space step, respectively. Based on the difference method, we obtain the following difference approximation:

$$
\begin{aligned}
& \left.\frac{\partial^{\alpha} u(x, t)}{\partial t^{\alpha}}\right|_{x_{i}, t_{k+1}} \\
& =\frac{1}{\Gamma(1-\alpha)} \sum_{j=0}^{k} \frac{u\left(x_{i}, t_{j+1}\right)-u\left(x_{i}, t_{j}\right)}{\tau} \int_{j \tau}^{(j+1) \tau} \frac{d \xi}{\left(t_{k+1}-\eta\right)^{\alpha}}+O(\tau) \\
& =\frac{\tau^{1-\alpha}}{\Gamma(2-\alpha)} \sum_{j=0}^{k} \frac{u\left(x_{i}, t_{k+1-j}\right)-u\left(x_{i}, t_{k-j}\right)}{\tau}\left[(j+1)^{1-\alpha}-j^{1-\alpha}\right]+O(\tau), \\
& \left.\frac{\partial^{\gamma} u(x, t)}{\partial x^{\gamma}}\right|_{x_{i}, t_{k}} \\
& =\frac{1}{\Gamma(1-\gamma)} \sum_{j=0}^{i-1} \frac{u\left(x_{j+1}, t_{k}\right)-u\left(x_{j}, t_{k}\right)}{h} \int_{j h}^{(j+1) h} \frac{d \eta}{\left(x_{i}-\zeta\right)^{\gamma}}+O(h) \\
& =\frac{h^{1-\gamma}}{\Gamma(2-\gamma)} \sum_{j=0}^{i-1} \frac{u\left(x_{i-j}, t_{k}\right)-u\left(x_{i-j-1}, t_{k}\right)}{h}\left[(j+1)^{1-\gamma}-j^{1-\gamma}\right]+O(h) .
\end{aligned}
$$

$\frac{\partial^{\beta} u(x, t)}{\partial x^{\beta}}$ uses the following improved Grünwald difference formula:

$$
\frac{\partial^{\beta} u(x, t)}{\partial x^{\beta}}=\frac{1}{h^{\beta}} \sum_{j=0}^{i+1} g_{j} u\left(x_{i+1-j}, t_{k}\right)+O(h),
$$

where $g_{0}=1, g_{1}=-\beta, g_{j-1}=(-1)^{j} \frac{\beta(\beta-1) \cdots(\beta-j+1)}{j !}$.

In the following, we will use the symbol regulation. Let

$$
\begin{aligned}
& u\left(x_{i}, t_{k}\right)=u_{i}^{k}, \quad s\left(x_{i}, t_{k}\right)=s_{i}^{k}, \quad a\left(x_{i}\right)=a_{i}, \quad b\left(x_{i}\right)=b_{i}, \\
& \varphi_{j}=(j+1)^{1-\alpha}-j^{1-\alpha}, \quad \omega_{j}=(j+1)^{1-\gamma}-j^{1-\gamma}, \\
& A_{i}=\frac{a_{i} \tau^{\alpha} \Gamma(2-\alpha)}{h^{\beta}}, \quad B_{i}=\frac{b_{i} \tau^{\alpha} \Gamma(2-\alpha)}{\Gamma(2-\gamma) h^{\gamma}} .
\end{aligned}
$$

Substituting the difference scheme (3.1)-(3.3) into the original equation (2.1) and the initial boundary conditions (2.2)-(2.3), we can obtain

$$
\begin{aligned}
\sum_{j=0}^{k} \varphi_{j}\left(u_{i}^{k+1-j}-u_{i}^{k-j}\right)= & -B_{i} \sum_{j=0}^{i-1} \omega_{j}\left(u_{i-j}^{k}-u_{i-j-1}^{k}\right)+A_{i} \sum_{j=0}^{i+1} u_{i+1-j}^{k} \\
& +\tau^{\alpha} \Gamma(2-\alpha) s_{i}^{k}+O(\tau+h) .
\end{aligned}
$$


When $k=0$,

$$
\begin{aligned}
u_{i}^{1}= & \left(1-B_{i}+g_{1} A_{i}\right) u_{i}^{0}-B_{i} \sum_{j=0}^{i-2}\left(\omega_{j+1}-\omega_{j}\right) u_{i-j-1}^{0} \\
& +A_{i} \sum_{j=0, \neq 1}^{i+1} g_{j} u_{i-j+1}^{0}+\tau^{\alpha} \Gamma(2-\alpha) s_{i}^{0} .
\end{aligned}
$$

When $k \geq 1$,

$$
\begin{aligned}
u_{i}^{k+1}= & \left(1-\varphi_{1}-B_{i}+g_{1} A_{i}\right) u_{i}^{k}+A_{i} \sum_{j=0, \neq 1}^{i+1} g_{j} u_{i-j+1}^{k}-B_{i} \sum_{j=0}^{i-2}\left(\omega_{j+1}-\omega_{j}\right) u_{i-j-1}^{k} \\
& -\sum_{j=1}^{k-1}\left(\varphi_{j+1}-\varphi_{j}\right) u_{i}^{k-j}+\varphi_{k} u_{i}^{0}+\tau^{\alpha} \Gamma(2-\alpha) s_{i}^{k} .
\end{aligned}
$$

\subsection{Stability analysis}

Let $\widetilde{u}_{i}^{k}, u_{i}^{k}(i=1,2, \ldots, m-1 ; k=1,2, \ldots, n-1)$ be the solutions of difference equation (3.4) which satisfy the initial conditions $f_{1}(x), f_{2}(x)$, respectively. Suppose that $s_{i}^{k}$ is accurate, the errors of the two solutions are defined as

$$
\varepsilon_{i}^{k}=\widetilde{u}_{i}^{k}-u_{i}^{k}
$$

If $k=0$, based on equation (3.5), we can get

$$
\varepsilon_{i}^{1}=\left(1-B_{i}+g_{1} A_{i}\right) \varepsilon_{i}^{0}-B_{i} \sum_{j=0}^{i-2}\left(\omega_{j+1}-\omega_{j}\right) \varepsilon_{i-j-1}^{0}+A_{i} \sum_{j=0, \neq 1}^{i+1} g_{j} \varepsilon_{i-j+1}^{0},
$$

where $i=1,2, \ldots, m-1$.

If $k \geq 1$, based on equation (3.6), it follows that

$$
\begin{aligned}
\varepsilon_{i}^{k+1}= & \left(1-\varphi_{1}-B_{i}+g_{1} A_{i}\right) \varepsilon_{i}^{k}+A_{i} \sum_{j=0, \neq 1}^{i+1} g_{j} \varepsilon_{i-j+1}^{k}-B_{i} \sum_{j=0}^{i-2}\left(\omega_{j+1}-\omega_{j}\right) \\
& * \varepsilon_{i-j-1}^{k}-\sum_{j=1}^{k-1}\left(\varphi_{j+1}-\varphi_{j}\right) \varepsilon_{i}^{k-j}+\varphi_{k} \varepsilon_{i}^{0},
\end{aligned}
$$

where $i=1,2, \ldots, m-1 ; k=1, \ldots, n-1$.

Let $E^{k}=\left[\varepsilon_{1}^{k}, \varepsilon_{2}^{k}, \ldots, \varepsilon_{m-1}^{k}\right]^{T}, \quad\left|\varepsilon_{L}^{k}\right|=\max _{1 \leq j \leq m-1}\left|\varepsilon_{j}^{k}\right|, \quad a_{q}=\max _{0 \leq i \leq m} a\left(x_{i}\right), \quad b_{q}=$ $\max _{0 \leq i \leq m} b\left(x_{i}\right)$, then we can get the following theorem.

Theorem 3.1 If $0<\frac{b_{q} \tau^{\alpha}}{h^{\gamma} \Gamma(2-\gamma)}+\frac{a_{q} \beta \tau^{\alpha}}{h^{\beta}} \leq \frac{2-2^{1-\alpha}}{\Gamma(2-\alpha)}$, then $\left\|E^{k}\right\|_{\infty} \leq\left\|E^{0}\right\|_{\infty}$, that is, the explicit difference schemes (3.8)-(3.9) are stable.

Proof Because $\sum_{j=0}^{N} g_{j}<0, \sum_{j=0, j \neq 1}^{N} g_{j}>0, \varphi_{j+1}-\varphi_{j}<0, \omega_{j+1}-\omega_{j}<0$, when $k=0$

$$
\left\|E^{1}\right\|_{\infty}=\left|\varepsilon_{L}^{1}\right| \leq\left[1-B_{i}-B_{i} \sum_{j=0}^{i-2}\left(\omega_{j+1}-\omega_{j}\right)+A_{i} \sum_{j=0}^{i+1} g_{j}\right]\left\|E^{0}\right\|_{\infty} \leq\left\|E^{0}\right\|_{\infty} .
$$


We use mathematical induction to prove it when $k \geq 1$. Suppose $\left\|E^{j}\right\|_{\infty} \leq\left\|E^{0}\right\|_{\infty}, j=$ $1,2, \ldots, k$, then

$$
\begin{aligned}
\left\|E^{k+1}\right\|_{\infty}= & \left|\varepsilon_{L}^{k+1}\right| \\
\leq & {\left[1-\varphi_{1}-B_{i}+A_{i} \sum_{j=0}^{i+1} g_{j}-B_{i} \sum_{j=0}^{i-2}\left(\omega_{j+1}-\omega_{j}\right)\right]\left\|E^{k}\right\|_{\infty} } \\
& -\sum_{j=1}^{k-1}\left(\varphi_{j+1}-\varphi_{j}\right)\left\|E^{k-j}\right\|_{\infty}+\varphi_{k}\left\|E^{0}\right\|_{\infty} \\
\leq & {\left[1+A_{i} \sum_{j=0}^{i+1} g_{j}\right]\left\|E^{0}\right\|_{\infty} \leq\left\|E^{0}\right\|_{\infty} . }
\end{aligned}
$$

So the difference scheme is stable. The theorem is proved.

\subsection{Convergence analysis}

Suppose that $u\left(x_{i}, t_{k}\right)$ is the exact solution of the differential equations (2.1)-(2.3) in the grid point $\left(x_{i}, t_{k}\right)$. Let $e_{i}^{k}=u\left(x_{i}, t_{k}\right)-u_{i}^{k}$ and $e_{k}=\left(e_{1}^{k}, e_{2}^{k}, \ldots, e_{m-1}^{k}\right)^{T}$. Then we have $u_{i}^{k}=u\left(x_{i}, t_{k}\right)-e_{i}^{k}, i=1,2, \ldots, m-1$.

Substituting difference equation and using $e^{0}=0$, we can the error iterative scheme. When $k=1$,

$$
e_{i}^{1}=R_{i, 1}, \quad i=1,2, \ldots, m-1
$$

When $k \geq 1$,

$$
\begin{aligned}
e_{i}^{k+1}= & \left(1-\varphi_{1}-B_{i}-g_{1} A_{i}\right) e_{i}^{k}+A_{i} \sum_{j=0, \neq 1}^{i+1} g_{j}-B_{i} \sum_{j=0}^{i-2}\left(\omega_{j+1}-\omega_{j}\right) \\
& * e_{i-j-1}^{k}-\sum_{j=1}^{k-1}\left(\varphi_{j+1}-\varphi_{j}\right) e_{i}^{k-j}+R_{i, k+1},
\end{aligned}
$$

where we use the solution $\left|R_{i, k+1}\right| \leq C\left(\tau^{1+\alpha}+\tau^{\alpha} h\right)$, and $i=1,2, \ldots, m-1 ; k=2, \ldots$, $n-1$.

Based on mathematical induction, we can get the following theorem.

Theorem 3.2 If $0<\frac{b_{q} \tau^{\alpha}}{h^{\gamma} \Gamma(2-\gamma)}+\frac{a_{q} \beta \tau^{\alpha}}{h^{\beta}} \leq \frac{2-2^{1-\alpha}}{\Gamma(2-\alpha)}$, then

$$
\left\|e^{k}\right\|_{\infty} \leq \varphi_{k-1}^{-1} C\left(\tau^{1+\alpha}+\tau^{\alpha} h\right), \quad k=1,2, \ldots, n .
$$

Proof Let $\left|e_{L}^{k}\right|=\max _{1 \leq i \leq m-1}\left|e_{i}^{k}\right|, k=1,2, \ldots, n$, we can get

$$
\left\|e^{1}\right\|_{\infty}=\left|e_{L}^{1}\right| \leq\left|R_{i, 1}\right| \leq b_{0}^{-1} C\left(\tau^{1+\alpha}+\tau^{\alpha} h\right) .
$$


Suppose $\left\|e^{j}\right\|_{\infty} \leq b_{j-1}^{-1} C\left(\tau^{1+\alpha}+\tau^{\alpha} h\right), j=1,2, \ldots, k$, because $g_{j}>g_{j+1}, \varphi_{j}>\varphi_{j+1}, j=$ $0,1,2, \ldots, n-1$, so we can obtain

$$
\begin{aligned}
\left\|e^{k+1}\right\|_{\infty}= & \left|e_{L}^{k+1}\right| \\
\leq & {\left[1-\varphi_{1}-B_{i}+A_{i} \sum_{j=0}^{i+1} g_{j}-B_{i} \sum_{j=0}^{i-2}\left(\omega_{j+1}-\omega_{j}\right)\right]\left\|e^{k}\right\|_{\infty} } \\
& -\sum_{j=1}^{k-1}\left(\varphi_{j+1}-\varphi_{j}\right)\left\|e^{k-j}\right\|_{\infty}+R_{i, k+1} \\
\leq & \left(1-\varphi_{1}-\sum_{j=1}^{k-1}\left(\varphi_{j+1}-\varphi_{j}\right)+\varphi_{k}\right) \\
& * \varphi_{k}^{-1} C\left(\tau^{1+\alpha}+\tau^{\alpha} h\right)=\varphi_{k}^{-1} C\left(\tau^{1+\alpha}+\tau^{\alpha} h\right) .
\end{aligned}
$$

So the theorem is proved.

Because $\lim _{k \rightarrow \infty} \frac{\varphi_{k}^{-1}}{k^{\alpha}}=\lim _{k \rightarrow \infty} \frac{k^{-\alpha}}{(k+1)^{1-\alpha}-k^{1-\alpha}}=\frac{1}{\alpha}$, then there is a constant $C>0$ that makes

$$
\left\|e^{k}\right\|_{\infty} \leq C k^{\alpha}\left(\tau^{1+\alpha}+\tau^{\alpha} h\right)=C(k \tau)^{\alpha}(\tau+h)
$$

If $k \tau<T$ is limited, we can get the following proposition easily.

Proposition 3.3 There is a constant $C_{1}=C T^{\alpha}$ such that

$$
\left\|u_{i}^{k}-u\left(x_{i}, t_{k}\right)\right\|_{\infty} \leq C_{1}(\tau+h), \quad i=1,2, \ldots, m-1 ; k=1,2, \ldots, n .
$$

By Theorems 3.1 and 3.2, we know that the explicit difference scheme has some strict conditions for the equation parameters and the time step and space step of the difference. To reduce this limit, we will discuss the implicit difference scheme.

\section{Implicit difference scheme}

Do the same mesh segmentation as the differential scheme shown above, and the following differential approximation for the equation is obtained:

$$
\begin{aligned}
\left.\frac{\partial^{\alpha} u(x, t)}{\partial t^{\alpha}}\right|_{x_{i}, t_{k+1}} & =\frac{1}{\Gamma(1-\alpha)} \sum_{j=0}^{k} \frac{u\left(x_{i}, t_{j+1}\right)-u\left(x_{i}, t_{j}\right)}{\tau} \int_{j \tau}^{(j+1) \tau} \frac{d \xi}{\left(t_{k+1}-\xi\right)^{\alpha}}+O(\tau) \\
= & \frac{\tau^{1-\alpha}}{\Gamma(2-\alpha)} \sum_{j=0}^{k} \frac{u\left(x_{i}, t_{k+1-j}\right)-u\left(x_{i}, t_{k-j}\right)}{\tau}\left[(j+1)^{1-\alpha}-j^{1-\alpha}\right]+O(\tau), \\
\left.\frac{\partial^{\gamma} u(x, t)}{\partial x^{\gamma}}\right|_{x_{i}, t_{k+1}}= & \frac{h^{1-\gamma}}{\Gamma(2-\gamma)} \sum_{j=0}^{i-1} \frac{u\left(x_{i-j}, t_{k+1}\right)-u\left(x_{i-j-1}, t_{k+1}\right)}{h} \\
& *\left[(j+1)^{1-\gamma}-j^{1-\gamma}\right]+O(h) .
\end{aligned}
$$


For $\frac{\partial^{\beta} u(x, t)}{\partial x^{\beta}}$ term, we replace it with the following Grünwald improved formula:

$$
\frac{\partial^{\beta} u\left(x_{i}, t_{k+1}\right)}{\partial x^{\beta}}=\frac{1}{h^{\beta}} \sum_{j=0}^{i+1} g_{j} u\left(x_{i+1-j}, t_{k+1}\right)+O(h)
$$

in which $g_{0}=1, g_{1}=-\beta, g_{j}=(-1)^{j} \frac{\beta(\beta-1) \cdots(\beta-j+1)}{j !}, j=1,2,3, \ldots$

Substituting the above difference equations into the original equation (2.1), we can obtain

$$
\begin{aligned}
& \frac{\tau^{1-\alpha}}{\Gamma(2-\alpha)} \sum_{j=0}^{k} \frac{u\left(x_{i}, t_{k+1-j}\right)-u\left(x_{i}, t_{k-j}\right)}{\tau}\left[(j+1)^{1-\alpha}-j^{1-\alpha}\right] \\
& =-b\left(x_{i}\right) \frac{h^{1-\gamma}}{\Gamma(2-\gamma)} \sum_{j=0}^{i-1} \frac{u\left(x_{i-j}, t_{k+1}\right)-u\left(x_{i-j-1}, t_{k+1}\right)}{h}\left[(j+1)^{1-\gamma}-j^{1-\gamma}\right] \\
& +\frac{a\left(x_{i}\right)}{h^{\beta}} \sum_{j=0}^{i+1} g_{j} u\left(x_{i-j+1}, t_{k+1}\right)+s\left(x_{i}, t_{k}\right)+O(\tau+h) .
\end{aligned}
$$

Using the same symbol mark as that in the last section, the difference equation (4.1) can be rewritten as

$$
\begin{aligned}
\sum_{j=0}^{k} \varphi_{j}\left(u_{i}^{k+1-j}-u_{i}^{k-j}\right)= & -B_{i} \sum_{j=0}^{i-1} \omega_{j}\left(u_{i-j}^{k+1}-u_{i-j-1}^{k+1}\right)+A_{i} \sum_{j=0}^{i+1} g_{j} u_{i+1-j}^{k+1} \\
& +\tau^{\alpha} \Gamma(2-\alpha) s_{i}^{k}, \quad k=0,1,2, \ldots, n .
\end{aligned}
$$

When $k=0$, by equation (4.2), we can get

$$
\begin{aligned}
(1 & \left.+B_{i} d_{0}-A_{i} g_{1}\right) u_{i}^{1}-A_{i} \sum_{j=0, \neq 1}^{i+1} g_{j} u_{i+1-j}^{1}+B_{i} \sum_{j=0}^{i-2}\left(\omega_{j+1}-\omega_{j}\right) u_{i-j-1}^{1}-B_{i} u_{0}^{1} \\
& =u_{i}^{0}+\tau^{\alpha} \Gamma(2-\alpha) s_{i}^{0} .
\end{aligned}
$$

When $k \geq 1$,

$$
\begin{aligned}
(1 & \left.+B_{i} d_{0}-A_{i} g_{1}\right) u_{i}^{k+1}-A_{i} \sum_{j=0, \neq 1}^{i+1} g_{j} u_{i+1-j}^{k+1}+B_{i} \sum_{j=0}^{i-2}\left(\omega_{j+1}-\omega_{j}\right) u_{i-j-1}^{k+1}-B_{i} u_{0}^{k+1} \\
& =\varphi_{k} u_{i}^{0}-\sum_{j=0}^{k-1}\left(\varphi_{j+1}-\varphi_{j}\right) u_{i}^{k-j}+\tau^{\alpha} \Gamma(2-\alpha) s_{i}^{k} .
\end{aligned}
$$

In the following, we will discuss the stability and convergence of the difference equations (4.3) and (4.4).

\subsection{Stability analysis}

Let $\widetilde{u}_{i}^{k}, u_{i}^{k}(i=1,2, \ldots, m-1 ; k=1,2, \ldots, n-1)$ be the solutions of equation (4.1), which satisfy the initial conditions $\tilde{f}(x), f(x)$, respectively. Suppose that the calculation of $s_{i}^{k}$ is 
accurate and the error $\varepsilon_{i}^{k}=\widetilde{u}_{i}^{k}-u_{i}^{k}$. By equations (4.3) and (4.4), the following equations are easy to get.

For $k=0$,

$$
\left(1+B_{i} d_{0}-A_{i} g_{1}\right) \varepsilon_{i}^{1}-A_{i} \sum_{j=0, \neq 1}^{i+1} g_{j} \varepsilon_{i+1-j}^{1}+B_{i} \sum_{j=0}^{i-2}\left(\omega_{j+1}-\omega_{j}\right) \varepsilon_{i-j-1}^{1}-B_{i} \varepsilon_{0}^{1}=\varepsilon_{i}^{0} .
$$

For $k \geq 1$,

$$
\begin{aligned}
& \left(1+B_{i} d_{0}-A_{i} g_{1}\right) \varepsilon_{i}^{k+1}-A_{i} \sum_{j=0, \neq 1}^{i+1} g_{j} \varepsilon_{i+1-j}^{k+1}+B_{i} \sum_{j=0}^{i-2}\left(\omega_{j+1}-\omega_{j}\right) \varepsilon_{i-j-1}^{k+1}-B_{i} \varepsilon_{0}^{k+1} \\
& =\varphi_{k} \varepsilon^{0}-\sum_{j=0}^{k-1}\left(\varphi_{j+1}-\varphi_{j}\right) \varepsilon_{i}^{k-j},
\end{aligned}
$$

where $i=1,2, \ldots, m-1 ; k=1, \ldots, n-1$.

Let $E^{k}=\left[\varepsilon_{1}^{k}, \varepsilon_{2}^{k}, \ldots, \varepsilon_{m-1}^{k}\right]^{T},\left|\varepsilon_{L}^{k}\right|=\max _{1 \leq j \leq m-1}\left|\varepsilon_{j}^{k}\right|, k=0,1,2, \ldots, n-1$. By mathematical induction, we can get the following theorem.

Theorem 4.1 For any $k \geq 0,\left\|E^{k}\right\|_{\infty} \leq\left\|E^{0}\right\|_{\infty}$, i.e., the implicit difference approximation defined is unconditionally stable.

Proof For $k=0$, by equation (4.5), we can get

$$
\begin{aligned}
\left\|E^{1}\right\|_{\infty} & =\left|\varepsilon_{L}^{1}\right| \leq\left|\left(1+B_{i} d_{0}-A_{i} g_{1}\right)-A_{i} \sum_{j=0, \neq 1}^{i+1} g_{j}+B_{i} \sum_{j=0}^{i-2}\left(\omega_{j+1}-\omega_{j}\right)-B_{i}\right| \\
& =\left|\varepsilon_{l}^{0}\right|=\left\|E^{0}\right\|_{\infty},
\end{aligned}
$$

where we use the result $\sum_{j=0}^{N} g_{j}<0$. Therefore, $\left\|E^{1}\right\|_{\infty} \leq\left\|E^{0}\right\|_{\infty}$.

Suppose $k \leq s$, we have $\left\|E^{k}\right\|_{\infty} \leq\left\|E^{0}\right\|_{\infty}, k=1,2, \ldots, s$. If $k=s+1$,

$$
\begin{aligned}
\left\|E^{s+1}\right\|_{\infty}= & \left|\varepsilon_{L}^{s+1}\right| \\
\leq & \left(1+B_{i} d_{0}-A_{i} g_{1}\right)\left|\varepsilon_{i}^{s+1}\right|-A_{i} \sum_{j=0, \neq 1}^{i+1} g_{j}\left|\varepsilon_{i+1-j}^{s+1}\right| \\
& +B_{i} \sum_{j=0}^{i-2}\left(\omega_{j+1}-\omega_{j}\right)\left|\varepsilon_{i-j-1}^{s+1}\right|-B_{i}\left|\varepsilon_{0}^{s+1}\right| \\
\leq & \mid\left(1+B_{i} d_{0}-A_{i} g_{1}\right) \varepsilon_{i}^{s+1}-A_{i} \sum_{j=0, \neq 1}^{i+1} g_{j} \varepsilon_{i+1-j}^{s+1} \\
& +B_{i} \sum_{j=0}^{i-2}\left(\omega_{j+1}-\omega_{j}\right) \varepsilon_{i-j-1}^{s+1}-B_{i} \varepsilon_{0}^{s+1} \mid
\end{aligned}
$$




$$
\begin{aligned}
& \leq\left|\varphi_{k} \varepsilon^{0}-\sum_{j=0}^{k-1}\left(\varphi_{j+1}-\varphi_{j}\right) \varepsilon_{i}^{k-j}\right| \\
& \leq\left|\varphi_{k}-\sum_{j=0}^{k-1}\left(\varphi_{j+1}-\varphi_{j}\right)\right|\left\|E^{0}\right\|_{\infty} \leq\left\|E^{0}\right\|_{\infty} .
\end{aligned}
$$

So, for any $k \geq 0$, we have $\left\|E^{k}\right\|_{\infty} \leq\left\|E^{0}\right\|_{\infty}$. Therefore, the implicit difference approximation is unconditionally stable. The proof is finished.

Compared to the explicit difference scheme, the implicit difference scheme for the original equation is unconditionally stable.

\subsection{Convergence analysis}

Suppose that $u\left(x_{i}, t_{k}\right)$ is the exact solution of the original equation at the grid point $\left(x_{i}, t_{k}\right)$. Let $e_{i}^{k}=u\left(x_{i}, t_{k}\right)-u_{i}^{k}$ and $e^{k}=\left(e_{1}^{k}, e_{2}^{k}, \ldots, e_{m-1}^{k}\right)$. Substituting the difference equations (4.3), (4.4) and using $e^{0}=0$, we can get, if $k=0$,

$$
\left(1+B_{i} d_{0}-A_{i} g_{1}\right) e_{i}^{1}-A_{i} \sum_{j=0, \neq 1}^{i+1} g_{j} e_{i+1-j}^{1}+B_{i} \sum_{j=0}^{i-2}\left(\omega_{j+1}-\omega_{j}\right) e_{i-j-1}^{1}-B_{i} e_{0}^{1}=R_{i}^{1} .
$$

If $k \geq 1$, we can get

$$
\begin{aligned}
(1 & \left.+B_{i} d_{0}-A_{i} g_{1}\right) e_{i}^{k+1}-A_{i} \sum_{j=0, \neq 1}^{i+1} g_{j} e_{i+1-j}^{k+1}+B_{i} \sum_{j=0}^{i-2}\left(\omega_{j+1}-\omega_{j}\right) e_{i-j-1}^{k+1}-B_{i} e_{0}^{k+1} \\
& =\varphi_{k} e_{i}^{0}-\sum_{j=0}^{k-1}\left(\varphi_{j+1}-\varphi_{j}\right) e_{i}^{k-j}+R_{i}^{k+1}
\end{aligned}
$$

where we use the solution $\left|R_{i}^{k+1}\right| \leq C\left(\tau^{1+\alpha}+\tau^{\alpha} h\right)$, where $C$ is a constant independent of $\tau$ and $h, i=1,2, \ldots, m-1 ; k=0,1,2, \ldots, n-1$. Below, by the mathematical induction method, we can prove the following theorem.

Theorem 4.2 For any $k=0,1,2, \ldots, n-1$, the error $\left\|e^{k}\right\|_{\infty} \leq \varphi_{k-1}^{-1} C\left(\tau^{1+\alpha}+\tau^{\alpha} h\right)$, where $\left\|e^{k}\right\|_{\infty}=\max _{1 \leq i \leq m-1}\left|e_{i}^{k}\right|$ and $C$ is constant.

Proof If $k=1$, suppose $\left|e_{L}^{1}\right|=\max _{1 \leq i \leq m-1}\left|e_{i}^{1}\right|$, then

$$
\begin{aligned}
\left\|e^{l}\right\|_{\infty}= & \left|e_{L}^{1}\right| \\
\leq & \left(1+B_{L} d_{0}-A_{L} g_{1}\right)\left|e_{L}^{1}\right|-A_{L} \sum_{j=0, \neq 1}^{L+1} g_{j}\left|e_{L+1-j}^{1}\right| \\
& +B_{L} \sum_{j=0}^{L-2}\left(\omega_{j+1}-\omega_{j}\right)\left|e_{L-j-1}^{1}\right|-B_{L}\left|e_{0}^{1}\right| \\
\leq & \mid\left(1+B_{L} d_{0}-A_{L} g_{1}\right) e_{L}^{1}-A_{L} \sum_{j=0, \neq 1}^{L+1} g_{j} e_{L+1-j}^{1}
\end{aligned}
$$




$$
\begin{gathered}
\quad+B_{L} \sum_{j=0}^{L-2}\left(\omega_{j+1}-\omega_{j}\right) e_{L-j-1}^{1}-B_{L} e_{0}^{1} \mid \\
=\left|R_{L}^{1}\right| \leq C\left(\tau^{1+\alpha}+\tau^{\alpha} h\right)=\varphi_{0}^{-1} C\left(\tau^{1+\alpha}+\tau^{\alpha} h\right),
\end{gathered}
$$

where $\varphi_{0}=1$.

Suppose $k \leq s$, we have $\left\|e^{k}\right\|_{\infty} \leq \varphi_{k-1}^{-1} C\left(\tau^{1+\alpha}+\tau^{\alpha} h\right), k=1,2, \ldots, s$. When $k=s+1$, suppose $\left|e_{L}^{s+1}\right|=\max _{1 \leq i \leq m-1}\left|e_{i}^{s+1}\right|$, then

$$
\begin{aligned}
\left\|e^{s+1}\right\|_{\infty}= & \left|e_{L}^{s+1}\right| \\
\leq & \varphi_{s}\left\|e^{0}\right\|_{\infty}-\sum_{j=0}^{s-1}\left(\varphi_{j+1}-\varphi_{j}\right)\left\|e^{s-j}\right\|_{\infty}+C\left(\tau^{1+\alpha}+\tau^{\alpha} h\right) \\
\leq & 0+\left(\varphi_{0}-\varphi_{1}\right) \varphi_{s-1}^{-1} C\left(\tau^{1+\alpha}+\tau^{\alpha} h\right)+\left(\varphi_{1}-\varphi_{2}\right) \varphi_{s-2}^{-1} \\
& * C\left(\tau^{1+\alpha}+\tau^{\alpha} h\right)+\cdots \\
& +\left(\varphi_{s-1}-\varphi_{s}\right) \varphi_{0}^{-1} C\left(\tau^{1+\alpha}+\tau^{\alpha} h\right)+C\left(\tau^{1+\alpha}+\tau^{\alpha} h\right) \\
\leq & {\left[\left(\varphi_{0}-\varphi_{1}\right)+\left(\varphi_{1}-\varphi_{2}\right)+\cdots+\left(\varphi_{s}-\varphi_{s}\right)\right] \varphi_{s}^{-1} C } \\
& *\left(\tau^{1+\alpha}+\tau^{\alpha} h\right)+C\left(\tau^{1+\alpha}+\tau^{\alpha} h\right) \\
= & \varphi_{s}^{-1} C\left(\tau^{1+\alpha}+\tau^{\alpha} h\right)
\end{aligned}
$$

where we use the solutions that $1>\varphi_{j}>\varphi_{i}>0$ for any $i>j$ and $\varphi_{j}^{-1} \leq \varphi_{s}^{-1}$ for $j=0,1,2, \ldots, s$. Therefore, the theorem is proved.

Because $\lim _{k \rightarrow \infty} \frac{\varphi_{k}^{-1}}{k^{\alpha}}=\lim _{k \rightarrow \infty} \frac{k^{-\alpha}}{(k+1)^{1-\alpha}-k^{1-\alpha}}=\frac{1}{\alpha}$, then there is a constant $C>0$ such that

$$
\left\|e^{k}\right\|_{\infty} \leq C k^{\alpha}\left(\tau^{1+\alpha}+\tau^{\alpha} h\right) .
$$

If $k \tau<T$ is limited, we can get the following proposition easily.

Proposition 4.3 There is a constant $C_{2}=C T^{\alpha}$ such that

$$
\left\|u_{i}^{k}-u\left(x_{i}, t_{k}\right)\right\|_{\infty} \leq C_{2}(\tau+h), \quad i=1,2, \ldots, m-1 ; k=1,2, \ldots, n .
$$

\section{Conclusions}

A time-space fractional advection diffusion equation is studied in this paper. Based on the difference method, an explicit difference scheme and an implicit difference scheme are obtained. Then we prove that the explicit difference scheme is conditionally stable and convergent and the implicit difference scheme is unconditionally stable and convergent. It is also obtained that the convergence order of the two methods is $O(\tau+h)$. The results obtained are a natural extension and generalization of the previous results, which can be used for reference in solving some fractional wave diffusion equations. 


\section{Funding}

This work was completed with the support of the National Natural Science Foundation of China (61603345), the

Outstanding Young Teacher Development Fund of Zhengzhou University (F0001175), the Key Scientific Research Project of Henan Higher Education (17A413003), and the General Financial Grant from the China Postdoctoral Science Foundation (2015M582202).

\section{Availability of data and materials}

Data sharing not applicable to this article as no datasets were generated or analysed during the current study.

\section{Competing interests}

The authors declare that there is no conflict of interest regarding the publication of this paper. The authors declare that they have no competing interests.

\section{Authors' contributions}

All authors read and approved the final manuscript.

\section{Publisher's Note}

Springer Nature remains neutral with regard to jurisdictional claims in published maps and institutional affiliations.

Received: 3 December 2018 Accepted: 2 January 2019 Published online: 18 January 2019

\section{References}

1. Barkai, E., Metzler, R., Klafter, J.: From continuous time random walks to the fractional Fokker-Planck equation. Phys. Rev. E 61, 132-138 (2000)

2. Chaves, A.: Fractional diffusion equation to describe Lévy flights. Phys. Lett. A 239, 13-16 (1998)

3. Gorenflo, R., Mainardi, F., Scalas, E., Raberto, M.: Fractional calculus and continuous-time finance. III. The diffusion limit. In: Mathematical Finance (Konstanz, 2000). Trends in Math., pp. 171-180. Birkhäuser, Basel (2001)

4. Ablowitz, M.J., Clarkson, P.A.: Solitons: Nonlinear Evolution Equations and Inverse Scattering. Cambridge University Press, Cambridge (1991)

5. Biler, P., Funaki, T., Woyczynski, W.A.: Fractal Burger equations. J. Differ. Equ. 148, 9-46 (1998)

6. Mann, J.A., Woyczynski, W.A.: Growing fractal interfaces in the presence of self-similar hopping surface diffusion. Physica A 291, 159-183 (2001)

7. Meerschaert, M., Tadjeran, C.: Finite difference approximations for two-sided space-fractional partial differential equations. Appl. Numer. Math. 56, 80-90 (2006)

8. Tan, C., Yang, L., Zhang, F., Zhang, Z.Q., Wong, W.S.: Stabilization of discrete time stochastic system with input delay and control dependent noise. Syst. Control Lett. 123, 62-68 (2019)

9. Ghiocel, G., Nicolae, P.: A numerical method for solving of the boundary value problems for ordinary differential equations. Results Math. 53, 295-302 (2009)

10. Zheng, B.: A new fractional Jacobi elliptic equation method for solving fractional partial differential equations. Adv. Differ. Equ. 2014, 228 (2014)

11. Yu, Q., Liu, F.: Implicit difference approximation for time fractional order reaction diffusion equation. J. Xiamen Univ. Natur. Sci. 45, 315-319 (2007)

12. Zhuang, P.: An explicit approximation for the space-time fractional diffusion equation. J. Comput. Math. Coll. Univ. 27, 223-228 (2005)

13. Tan, P., Zhang, X.: A numerical method for the space-time fractional convection-diffusion equation. Math. Numer. Sin. 30, 305-310 (2008)

14. Povstenko, Y.: Solutions to time-fractional diffusion-wave equation in cylindrical coordinates. Adv. Differ. Equ. 2011, Article ID 930297 (2011)

15. Zhang, F.F., Jiang, X.Y.: Analytical solutions for a time-fractional axisymmetric diffusion-wave equation with a source term. Nonlinear Anal., Real World Appl. 12(3), 1841-1849 (2011)

16. Parvizi, M., Eslahchi, M.R., Dehghan, M.: Numerical solution of fractional advection-diffusion equation with a nonlinear source term. Numer. Algorithms 68, 601-629 (2015)

17. Bu, W., Liu, X., Tang, Y., Jiang, Y.: Finite element multigrid method for multi-term time fractional advection-diffusion equations. Int. J. Model. Simul. Sci. Comput. 6(1), 1540001 (2015)

18. Povstenko, Y., Kyrylych, T.: Two approaches to obtaining the space-time fractional advection-diffusion equation. Entropy 19(7), 297 (2017)

19. Mohyud-DinEmail, S., Akram, T., Abbas, M., Ismail, A., Ali, N.: A fully implicit finite difference scheme based on extended cubic B-splines for time fractional advection-diffusion equation. Adv. Differ. Equ. 2018, 109 (2018)

20. Marin, M., Andreas, Ö.: The effect of a dipolar structure on the Holder stability in Green-Naghdi thermoelasticity. Contin. Mech. Thermodyn. 29(6), 1365-1374 (2017)

21. Donatelli, M., Mazza, M., Serra-Capizzano, S.: Spectral analysis and structure preserving preconditioners for fractional diffusion equation. J. Comput. Phys. 307, 262-279 (2016)

22. Lin, X.L., Ng, M.K., Sun, H.W.: A multigrid method for linear systems arising from time-dependent two-dimensional space-fractional diffusion equations. J. Comput. Phys. 336, 69-86 (2017) 